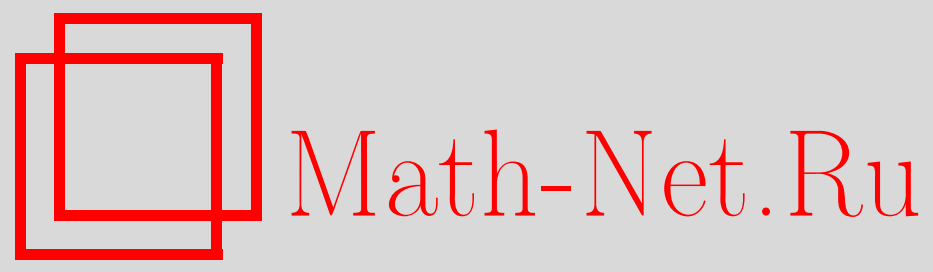

О. В. Кузьмин, О. В. Леонова, Об аналитической сопряженности полиномов Тушара и им квазиортогональных, Дискрет. матем., 2002, том 14, выпуск 1, 151-157

DOI: https://doi.org/10.4213/dm228

Использование Общероссийского математического портала Math-Net.Ru подразумевает, что вы прочитали и согласны с пользовательским соглашением http: //www.mathnet.ru/rus/agreement

Параметры загрузки:

IP: 3.91 .87 .62

26 апреля 2023 г., 15:12:36

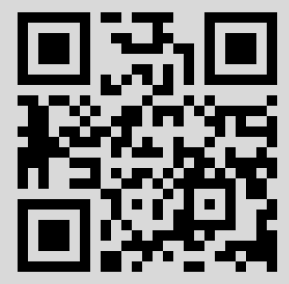


УДК 519.15

\title{
Об аналитической сопряженности полиномов Тушара и им квазиортогональных
}

\author{
(c) 2002 г. О. В. Кузьмин, О. В. Леонова
}

\begin{abstract}
Рассматриваются полиномы Тушара, являющиеся обобщениями полиномов Белла, и $P$-полиномы, составляющие с ними квазиортогональную систему. Установлена аналитическая сопряженность полиномов Тушара и $P$-полиномов, что позволило, в частности, найти для последних ряд новых рекуррентных соотношений.
\end{abstract}

\section{1. Введение}

В связи с изучением некоторых циклических подстановок Тушар в [1] ввел ряд обобщений полиномов Белла. Для одного из таких обобщений $T_{n, k}(x, y)$, названного полиномами Тушара, в [2] получены экспоненциальные производящие функции и рекуррентные соотношения.

В $[3,4]$ авторами данной публикации изучаются полиномы $T_{n, k}(x, y)$, при этом найдены некоторые свойства, перечислительные интерпретации и вероятностные приложения. В [3] вводятся новые, так называемые $P$-полиномы, составляющие с полиномами Тушара квазиортогональную систему. Для $P$-полиномов $P_{n, k}(x, y)$ получено явное выражение, производящие функции и рекуррентные соотношения, построены алгоритм преобразования полиномов Тушара в $P$-полиномы и ему обратный.

Во втором параграфе статьи приводятся необходимые определения и свойства полиномов Белла и Платонова.

В третьем параграфе приводятся необходимые определения и свойства полиномов Тушара и $P$-полиномов.

В четвертом параграфе установлена аналитическая сопряженность полиномов Тушара и $P$-полиномов.

В пятом параграфе получены новые рекуррентные соотношения.

\section{2. Полиномы Белла и Платонова}

Однородные полиномы Белла от переменных $x_{i}, i \geqslant 1$, которые мы будем обозначать $A_{n, k}(x)=A\left(n, k ; x_{1}, x_{2}, \ldots\right), 1 \leqslant k \leqslant n$, и в дальнейшем называть $A$-полиномами, впервые рассматривались Беллом. Пусть последовательность аргументов 
$A$-полиномов задана в виде производящей функции

$$
x(t)=\sum_{n=1}^{\infty} x_{n} \frac{t^{n}}{n !} .
$$

Производящими функциями $A$-полиномов являются

$$
\sum_{n=k}^{\infty} A_{n, k}(x) \frac{t^{n}}{n !}=\frac{(x(t))^{k}}{k !}
$$

Из (1) может быть получена явная формула для $A$-полиномов

$$
A_{n, k}(x)=n ! \sum_{n, k} \prod_{i=1}^{n-k+1} x_{i}^{r_{i}}\left[r_{i} !(i !)^{r_{i}}\right]^{-1}
$$

где $A_{0,0}(x)=1$ и суммирование ведется по всем $r_{i} \geqslant 0,1 \leqslant i \leqslant n$, таким, что

$$
\sum_{i=1}^{n-k+1} r_{i}=k, \quad \sum_{i=1}^{n-k+1} i r_{i}=n .
$$

Известно [5], что если $F(t)=f(u), u=g(t)$, то

$$
A_{k, r}(F)=\sum_{s=r}^{k} A_{k, s}(g) A_{s, r}(f), \quad 1 \leqslant r \leqslant k .
$$

C $A$-полиномами тесно связаны $B$-полиномы Платонова (см. [5])

$$
B_{n, k}(x)=\frac{(-1)^{n-k}}{(k-1) ! x_{1}^{2 n-k}} \sum_{\substack{2 n-2 k \\ n-k}}(-1)^{r_{1}} r_{1} !\left(2 n-k-r_{1}-1\right) ! \prod_{i=1}^{n-k+1} x_{i}^{r_{i}}\left(r_{i} !(i !)^{r_{i}}\right)^{-1},
$$

где $n \geqslant 2,1 \leqslant k \leqslant n-1, x_{1} \neq 0, B_{n, n}=x_{1}{ }^{-n}$ при $n \geqslant 0$ и суммирование ведется по всем $r_{i} \geqslant 0,1 \leqslant i \leqslant 2 n-2 k$ таким, что

$$
\sum_{i=1}^{n-k+1} r_{i}=n-k, \quad \sum_{i=1}^{n-k+1} i r_{i}=2 n-2 k .
$$

Тесная связь $A$ - и $B$-полиномов объясняется тем, что они двояко, алгебраически и аналитически, сопряжены. Алгебраическая сопряженность определяется взаимной обратностью бесконечных нижних треугольных матриц $\left\|A_{n, k}(x)\right\|$ и $\left\|B_{n, k}(x)\right\|$. Аналитическая сопряженность выражается системой тождеств

$$
A_{n, k}(x)=B_{n, k}(\tilde{x})
$$

где $x(t)$ и $\tilde{x}(u)$ - взаимно-обратные функции, то есть

$$
x(\tilde{x}(u))=u, \quad \tilde{x}(x(t))=t .
$$

Дополнительно полагаем $A_{0, k}(x)=B_{0, k}(x)=\delta_{0, k}$, где $\delta_{n, k}$ - символ Кронекера, $A_{n, k}(x)=B_{n, k}(x)=0$, если $k \notin\{1,2, \ldots, n\}$. 
С учетом соотношения (5) из (1) получаем, что производящими функциями для $B$-полиномов являются

$$
\sum_{n=k}^{\infty} B_{n, k}(x) \frac{u^{n}}{n !}=\frac{(\tilde{x}(u))^{k}}{k !}
$$

Для $B$-полиномов справедливы рекуррентные соотношения

$$
\begin{aligned}
\left(\begin{array}{c}
k \\
r
\end{array}\right) B_{n, k}(x) & =\sum_{i=0}^{n-k}\left(\begin{array}{c}
n \\
r+i
\end{array}\right) B_{r+i, r}(x) B_{n-r-i, k-r}(x), \quad 1 \leqslant r \leqslant k \leqslant n, \\
\frac{\partial}{\partial x_{i}} B_{n, k}(x) & =-\left(\begin{array}{c}
k+i-1 \\
i
\end{array}\right) B_{n+1, k+i}(x), \quad k, i \geqslant 1, \quad k+i \leqslant n+1,
\end{aligned}
$$

полученные в [6] и [7] соответственно.

\section{3. Полиномы Тушара и $P$-полиномы}

Полиномы от переменных $x_{i}$ и $y_{i}, i \geqslant 1$, которые мы будем обозначать

$$
T_{n, k}(x, y)=T_{n, k}\left(x_{1}, \ldots ; y_{1}, \ldots\right)
$$

для $n, k \geqslant 1, k \leqslant n$, и в дальнейшем называть полиномами Тушара, впервые рассматривались Тушаром в [1]. Производящая функция полиномов Тушара имеет вид

$$
\sum_{n=k}^{\infty} T_{n, k}(x, y) \frac{t^{n}}{n !}=(x(t))^{k} \frac{e^{y(t)}}{k !}
$$

где

$$
x(t)=\sum_{i=1}^{\infty} x_{i} \frac{t^{i}}{i !}, \quad y(t)=\sum_{i=1}^{\infty} y_{i} \frac{t^{i}}{i !} .
$$

Известен явный вид этих полиномов (см., например, [2]):

$$
T_{n, k}(x, y)=n ! \sum_{(n ; k)} \prod_{i=1}^{n} x_{i}^{r_{i}} y_{i}^{s_{i}}\left(r_{i} ! s_{i} !(i !)^{r_{i}+s_{i}}\right)^{-1}
$$

где $T_{0,0}(x, y)=1$ и суммирование ведется по всем $r_{i}, s_{i} \geqslant 0,1 \leqslant i \leqslant n$, таким, что

$$
\sum_{i=1}^{n} r_{i}=k, \quad \sum_{i=1}^{n} i\left(r_{i}+s_{i}\right)=n .
$$

Непосредственно из (10) следует, что $T_{n, 0}(x, y)=Y_{n}(y)$, где $Y_{n}(y)=Y_{n}\left(y_{1}, \ldots, y_{n}\right)$ - известные полиномы Белла, и $T_{n, n}(x, y)=x_{1}^{n}=A_{n n}(x)$. Из сопоставления формул (1) и (9) или (2) и (10) видно, что $T_{n, k}(x, 0)=A_{n, k}(x)$.

Для полиномов Тушара справедливы (см. [2]) соотношения

$$
\begin{aligned}
k T_{n, k}(x, y) & =\sum_{r=k-1}^{n}\left(\begin{array}{l}
n \\
r
\end{array}\right) x_{n-r} T_{r, k-1}(x, y), \\
T_{n, k}(x, y) & =\sum_{i=0}^{n}\left(\begin{array}{l}
n \\
i
\end{array}\right)\left(x_{i+1} T_{n-i, k-1}(x, y)+y_{i+1} T_{n-i, k}(x, y)\right) .
\end{aligned}
$$


Полиномы Тушара связаны с $A$ - и $B$-полиномами равенством [3]

$$
T_{n, k}(x, y)=\sum_{j=k}^{n}\left(\begin{array}{l}
n \\
j
\end{array}\right) A_{j, k}(x) \sum_{p=0}^{n-j} A_{n-j, p}(y) .
$$

$P$-полиномы можно определить, задав их производящую функцию [3]

$$
\sum_{n=k}^{\infty} P_{n, k}(x, y) \frac{u^{n}}{n !}=\frac{(\tilde{x}(u))^{k}}{k !} e^{-y(\tilde{x}(u))}, \quad k \geqslant 0 .
$$

Разлагая в ряд производящую функцию (14) и приравнивая коэффициенты при $u^{n} / n$ !, получаем явную формулу для $P$-полиномов

$$
\begin{aligned}
P_{n, k}(x, y)=\frac{(-1)^{n-k}}{k ! x_{1}^{2 n-k}} & \sum_{(2 n-2 k, n-k)}(-1)^{r_{1}+s_{1}+s_{2} \ldots+s_{n}} r_{1} !\left(2 n-k-r_{1}-1\right) ! \\
& \times\left(\sum_{i=1}^{n} i s_{i}+k\right) \prod_{i=1}^{n} x_{i}^{r_{i}} y_{i}^{s_{i}}\left(r_{i} ! s_{i} !(i !)^{r_{i}+s_{i}}\right)^{-1}, \quad 1 \leqslant k \leqslant n,
\end{aligned}
$$

где $x_{1} \neq 0, P_{n, n}(x, y)=x_{1}{ }^{-n}, n \geqslant 1$ и суммирование ведется по всем $r_{i}, s_{i} \geqslant 0$, $1 \leqslant i \leqslant n$, таким, что

$$
\sum_{i=1}^{n} r_{i}=n-k, \quad \sum_{i=1}^{n} i\left(r_{i}+s_{i}\right)=2 n-2 k .
$$

Из сопоставления формул (3) и (6) видно, что $P_{n, k}(x, 0)=B_{n, k}(x)$, то есть $P$ полиномы являются обобщениями для $B$-полиномов Платонова.

Как показано в [3], $P$-полиномы выражаются через $A$ - и $B$-полиномы формулой

$$
P_{n, k}(x, y)=\sum_{j=k}^{n}\left(\begin{array}{l}
j \\
k
\end{array}\right) B_{n, j}(x) \sum_{p=0}^{j-k}(-1)^{p} A_{j-k, p}(y)
$$

и связаны с полиномами Тушара соотношениями квазиортогональности

$$
\begin{aligned}
& \sum_{i=k}^{n} T_{n, i}(x, y) P_{i, k}(x, y)=\delta_{n, k}, \\
& \sum_{i=k}^{n} P_{n, i}(x, y) T_{i, k}(x, y)=\delta_{n, k}, \quad 1 \leqslant k \leqslant n .
\end{aligned}
$$

\section{4. Аналитическая сопряженность}

Теорема 1. Полиномъ Тушара и Р-полиномы удовлетворяют соотношениям

$$
T_{n, k}(\tilde{x}, \bar{y})=P_{n, k}(x, y),
$$

где функции $x(t)$ и $\tilde{x}(u)$ взаимно-обратны, а $\bar{y}(u)=-y(\tilde{x}(u))$. 
Доказательство. Используя соотношения (13) и (5), получим

$$
\begin{aligned}
T_{n, k}(\tilde{x}, \bar{y}) & \left.=A_{n, k}(\tilde{x})+\sum_{j=k}^{n-1}\left(\begin{array}{c}
n \\
j
\end{array}\right) A_{j, k}(\tilde{x}) \sum_{p=1}^{n-j} A_{n-j, p}(\bar{y})\right) \\
& =B_{n, k}(x)+\sum_{j=k}^{n-1}\left(\begin{array}{c}
n \\
j
\end{array}\right) B_{j, k}(x) \sum_{p=1}^{n-j} A_{n-j, p}(-y(\tilde{x})) \\
& =B_{n, k}(x)+\sum_{j=k}^{n-1}\left(\begin{array}{c}
n \\
j
\end{array}\right) B_{j, k}(x) \sum_{p=1}^{n-j}(-1)^{p} A_{n-j, p}(y(\tilde{x}))
\end{aligned}
$$

Из последнего соотношения с учетом (3) находим, что

$$
\begin{aligned}
T_{n, k}(\tilde{x}, \bar{y}) & =B_{n, k}(x)+\sum_{j=k}^{n-1}\left(\begin{array}{c}
n \\
j
\end{array}\right) B_{j, k}(x) \sum_{p=1}^{n-j}(-1)^{p} \sum_{s=p}^{n-j} A_{n-j, s}(\tilde{x}) A_{s, p}(y) \\
& =B_{n, k}(x)+\sum_{j=k}^{n-1} \sum_{p=1}^{n-j} \sum_{s=p}^{n-j}\left(\begin{array}{c}
n \\
j
\end{array}\right) B_{j, k}(x)(-1)^{p} B_{n-j, s}(x) A_{s, p}(y) \\
& =B_{n, k}(x)+\sum_{p=1}^{n-k} \sum_{s=p}^{n-k} \sum_{j=k}^{n-s}\left(\begin{array}{c}
n \\
j
\end{array}\right) B_{j, k}(x) B_{n-j, s}(x)(-1)^{p} A_{s, p}(y) \\
& =B_{n, k}(x)+\sum_{p=1}^{n-k} \sum_{s=p}^{n-k}(-1)^{p} A_{s, p}(y) \sum_{j=k}^{n-s}\left(\begin{array}{c}
n \\
j
\end{array}\right) B_{j, k}(x) B_{n-j, s}(x) .
\end{aligned}
$$

Из последнего равенства и соотношений (7) и (15) получаем, что

$$
\begin{aligned}
T_{n, k}(\tilde{x}, \bar{y}) & =B_{n, k}(x)+\sum_{p=1}^{n-k} \sum_{s=p}^{n-k}(-1)^{p} A_{s, p}(y)\left(\begin{array}{c}
k+s \\
s
\end{array}\right) B_{n, k+s}(x) \\
& =B_{n, k}(x)+\sum_{p=1}^{n-k} \sum_{i=p+k}^{n}(-1)^{p} A_{i-k, p}(y)\left(\begin{array}{c}
i \\
k
\end{array}\right) B_{n, i}(x) \\
& =B_{n, k}(x)+\sum_{i=k+1}^{n} \sum_{p=1}^{i-k}(-1)^{p} A_{i-k, p}(y)\left(\begin{array}{l}
i \\
k
\end{array}\right) B_{n, i}(x) \\
& =\sum_{i=k}^{n}\left(\begin{array}{l}
i \\
k
\end{array}\right) B_{n, i}(x) \sum_{p=0}^{i-k}(-1)^{p} A_{i-k, p}(y)=P_{n, k}(x, y)
\end{aligned}
$$

что и дает соотношение (16). Теорема доказана.

\section{5. Рекуррентные соотношения}

Теорема 2. Для P-полиномов справедливы соотношения

$$
\frac{\partial}{\partial x_{i}} P_{n, k}(x, y)=-\left(\begin{array}{c}
k+i \\
k
\end{array}\right)\left(P_{n+1, k+i}(x, y)-i \tilde{P}_{n+1, k+i}(x, y)\right),
$$


əde

$$
\tilde{P}_{n, k}(x, y)=\sum_{j=k}^{n} \frac{1}{j}\left(\begin{array}{l}
j \\
k
\end{array}\right) B_{n, j}(x) \sum_{p=0}^{j-k}(-1)^{p} A_{j-k, p}(y)
$$

Доказательство. Продифференцировав соотношение (15) по $x_{i}$, с учетом (8), получим, что

$$
\begin{aligned}
& \frac{\partial}{\partial x_{i}} P_{n, k}(x, y)=\frac{\partial}{\partial x_{i}} \sum_{j=k}^{n}\left(\begin{array}{l}
j \\
k
\end{array}\right) B_{n, j}(x) \sum_{p=0}^{j-k}(-1)^{p} A_{j-k, p}(y) \\
& =-\sum_{j=k}^{n}\left(\begin{array}{l}
j \\
k
\end{array}\right)\left(\begin{array}{c}
i+j-1 \\
i
\end{array}\right) B_{n+1, j+i}(x) \sum_{p=0}^{j-k}(-1)^{p} A_{j-k, p}(y) \\
& =-\sum_{j=k+i}^{n+i}\left(\begin{array}{c}
j-i \\
k
\end{array}\right)\left(\begin{array}{c}
j-1 \\
i
\end{array}\right) B_{n+1, j}(x) \sum_{p=0}^{j-i-k}(-1)^{p} A_{j-i-k, p}(y) \\
& =-\left(\begin{array}{c}
k+i \\
k
\end{array}\right) \sum_{j=k+i}^{n+1} \frac{j-i}{j}\left(\begin{array}{c}
j \\
k+i
\end{array}\right) B_{n+1, j}(x) \sum_{p=0}^{j-i-k}(-1)^{p} A_{j-i-k, p}(y) \\
& =-\left(\begin{array}{c}
k+i \\
k
\end{array}\right)\left(\sum_{j=k+i}^{n+1}\left(\begin{array}{c}
j \\
k+i
\end{array}\right) B_{n+1, j}(x) \sum_{p=0}^{j-i-k}(-1)^{p} A_{j-i-k, p}(y)-\right. \\
& \left.-\sum_{j=k+i}^{n+1} \frac{i}{j}\left(\begin{array}{c}
j \\
k+i
\end{array}\right) B_{n+1, j}(x) \sum_{p=0}^{j-i-k}(-1)^{p} A_{j-i-k, p}(y)\right) \\
& =-\left(\begin{array}{c}
k+i \\
k
\end{array}\right)\left(P_{n+1, k+i}(x, y)-i \tilde{P}_{n+1, k+i}(x, y)\right) \text {, }
\end{aligned}
$$

где

$$
\tilde{P}_{n, k}(x, y)=\sum_{j=k}^{n} \frac{1}{j}\left(\begin{array}{l}
j \\
k
\end{array}\right) B_{n, j}(x) \sum_{p=0}^{j-k}(-1)^{p} A_{j-k, p}(y)
$$

Теорема доказана.

Равенство (16) позволяет получить ряд новых соотношений для $P$-полиномов.

Непосредственно из соотношений (5), (11) и (16) получаем, что

$$
k P_{n, k}(x, y)=\sum_{r=k-1}^{n}\left(\begin{array}{l}
n \\
r
\end{array}\right) B_{n-r, 1}(x) P_{r, k-1}(x, y)
$$


Из соотношений $(5),(12)$ и (16) следует, что

$$
\begin{aligned}
P_{n, k}(x, y) & =T_{n, k}(\tilde{x}, \bar{y})=\sum_{i=0}^{n}\left(\begin{array}{l}
n \\
i
\end{array}\right)\left(\tilde{x}_{i+1} T_{n-i, k-1}(\tilde{x}, \bar{y})+\bar{y}_{i+1} T_{n-i, k}(\tilde{x}, \bar{y})\right) \\
& =\sum_{i=0}^{n}\left(\begin{array}{c}
n \\
i
\end{array}\right)\left(B_{i+1,1}(x) P_{n-i, k-1}(x, y)+\bar{y}_{i+1} P_{n-i, k}(x, y)\right) \\
& =\sum_{i=0}^{n}\left(\begin{array}{c}
n \\
i
\end{array}\right)\left(B_{i+1,1}(x) P_{n-i, k-1}(x, y)-\left(\sum_{j=1}^{i+1} y_{j} B_{i+1, j}(x)\right) P_{n-i, k}(x, y)\right) .
\end{aligned}
$$

\section{Список литературы}

1. Touchard J., Sur les cycles des substitutious. Acta Math. (1939) 70, 243-279.

2. Chrysaphinou O., On Touchard polynomials. Discrete Math. (1985) 54, 143-152.

3. Кузьмин О. В., Леонова О. В., Полиномы Тушара и им квазиортогональные. Оптимизачия, управлеяие, иятеллект. ИДСТУ СО РАН, Иркутск, 1999, N3, 218-227.

4. Кузьмин О. В., Леонова О. В., Полиномы Тушара и их приложения. Дискретная математика (2000) 12, Ne3, 60-71.

5. Платонов М. Л., Комбинаторнъе числа класса отображений и их приложения. Наука, Москва, 1979.

6. Платонов М. Л., Комбинаторные полиномы в алгебре операторов, перестановочных со сдвигом. Дискретная математиха (1992) 4, N1, 33-49.

7. Кузьмин О. В., Рекуррентные соотношения и перечислительные интерпретации некоторых комбинаторных чисел и полиномов. Дискретная математиха (1994) 6, №3, $39-49$.

Статья поступила 08.11.2001. 